\title{
The Combined Internal and Principal Parametric Resonances on Continuum Stator System of Asynchronous Machine
}

\author{
Baizhou Li ${ }^{1,2}$ and Qichang Zhang \\ ${ }^{1}$ School of Mechanical Engineering, Tianjin University, Tianjin 300072, China \\ ${ }^{2}$ Tianjin Key Laboratory of High Cutting and Precision Machining, Tianjin University of Technology and Education, \\ Tianjin 300222, China \\ Correspondence should be addressed to Baizhou Li; bzli1024@tju.edu.cn
}

Received 31 March 2014; Accepted 30 July 2014; Published 22 December 2014

Academic Editor: Dumitru I. Caruntu

Copyright (C) 2014 B. Li and Q. Zhang. This is an open access article distributed under the Creative Commons Attribution License, which permits unrestricted use, distribution, and reproduction in any medium, provided the original work is properly cited.

\begin{abstract}
With the increasing requirement of quiet electrical machines in the civil and defense industry, it is very significant and necessary to predict the vibration and noise characteristics of stator and rotor in the early conceptual phase. Therefore, the combined internal and principal parametric resonances of a stator system excited by radial electromagnetic force are presented in this paper. The stator structure is modeled as a continuum double-shell system which is loaded by a varying distributed electromagnetic load. The nonlinear dynamic equations are derived and solved by the method of multiple scales. The influences of mechanical and electromagnetic parameters on resonance characteristics are illustrated by the frequency-response curves. Furthermore, the RungeKutta method is adopted to numerically analyze steady-state response for the further understanding of the resonance characteristics with different parameters.
\end{abstract}

\section{Introduction}

As the electromagnetic conversion device between mechanical and electrical energy, electrical machines are widely used in the civil and defense industry. The electromagnetic vibration of the stator system, which is one of the main noise sources, can directly affect the noise level of electrical machines. Therefore, with the development of quiet electrical machines, it is very significant to study electromagnetic vibration mechanism of the stator system for noise reduction.

Consideration of the levels of vibration and noise at the design stage has been of importance for many years, and it is still a major consideration for machine designers and operators. It is well known that vibration and noise can be reduced to a very large extent when the electromagnetic force is not allowed to excite any kind of resonances of electrical machines. In fact, the electromagnetic load still contains many frequency components and it will excite radial vibrations of the stator system and generate strong noise. Moreover, the resonance responses of the stator system make the electromagnetic load change by affecting the air-gap magnetic field. Therefore, the interaction mechanism between mechanical and electromagnetic behavior is of great concern in this paper.

Many researches have been developed in electromagnetic vibration on the stator system of multifarious electrical machines over the years in the analytical and the numerical methods. Some of them pay attention to establishing the models of stator and rotor and calculating the natural characteristics by finite element method (FEM) which could provide accurate solutions for dynamic system with complicated stator and rotor shapes [1-5]. The purpose is to achieve lower levels of vibrations by designing the electromagnetic force to have excitation frequencies away from natural frequencies of stator and rotor. And the models have been developed from rigid bodies to elastic bodies. However, some of the researchers studied the origins of electromagnetic excitation which are the Maxwell forces, the Lorentz forces, and the magnetostrictive forces $[6,7]$. The Maxwell forces are the predominant forces that act on the boundary regions from the air-gap to the stator core [8-12]. In particular, Pennacchi and Frosini presented a model to calculate the magnetic pull 
for the dynamical behavior analysis of large-size generators [9]. And the method proposed in [9] has been validated using experimental results in [10]. Furthermore, nonlinear effects due to electromechanical interaction have been analyzed based on the proposed method in [11]. Therefore, the analysis of the vibration characteristics of the stator system under Maxwell forces is very essential.

On the other hand, for the continuum vibration, Qing et al. [13] analyzed the natural frequencies and mode shapes by a semianalytical method based on thick double-shell systems. Established a continuum beam model for the nonlinear vibration of rotor-bearing system. Nguyen [14] and Hegazy [15] presented the nonlinear resonances involving two mode shapes of thin plates. It has been shown that when a parametric resonance is excited in the presence of an internal resonance, the coincidence of these two types of resonances will give rise to the simultaneous resonances.

In spite of publications on the electromagnetic vibration of the electrical machines, very few of them focused on the continuum stator system under the coupling electromagnetic forces between the electromagnetic and displacement fields. In this paper, the complicated stator system of a three-phase asynchronous machine is simplified to a continuous elastic structure with two coaxial cylindrical shells. The electromagnetic force expression with the effect of resonance response of the stator system is given. Based on the energy principle the nonlinear dynamic equation is derived. And the method of multiple scales is employed to develop the nonlinear dynamic analysis of the continuous system. The jump phenomenon is pointed out, and the influences of detuning parameters, damping coefficients, and electromagnetic parameters are illustrated by the frequency-response curves. The steady-state responses of the combined internal and principal parametric resonances are obtained with the Runge-Kutta method. Diagrams of time history, phase chart, the fast Fourier transformation (FFT), and Poincaré section are presented to confirm both the effects of different parameters and the response of the system.

\section{Distributed Electromagnetic Forces}

The electromagnetic force contains radial and tangential components. The radial component can excite the radial vibration of the stator core, and it is the main source of the electromagnetic noise. The tangential component, which is the reaction force corresponding to the electromagnetic torque, bends the tooth root, and it is the minor source of the electromagnetic noise. Therefore, it is generally accepted that the tangential component is negligible in the analysis of radial vibration. The radial electromagnetic force acts on the stator core and generates the radial displacement $w_{s}$ of the stator core. Meanwhile, the radial displacement changes the thickness of the air-gap and then changes the air-gap permeance $\Lambda$ as

$$
\Lambda(x, \theta)=\frac{\Lambda_{0}}{1+W}=\Lambda_{0}\left(1-W+W^{2}-W^{3}+W^{4}+\cdots\right),
$$

where $\Lambda_{0}$ is the average air-gap permeance. $W=w_{s} / \delta_{0}$ is the dimensionless radial displacement of the stator core, and $\delta_{0}$ is the average eccentricity. It is well known that the dynamic eccentricity and mechanical imbalance can produce the rotational frequency vibrations. But the eccentricity is neglected because it merely modifies the expression of (1) and will not change the mechanism of producing vibrations. Then, the radial electromagnetic force [8-12] is proportional to the square of the magnetic flux-density which is the product of the magnetomotive force and air-gap permeance, and it can be derived as

$$
\begin{aligned}
q_{r}= & Z_{0}+Z_{1} w_{s}+Z_{2} w_{s}^{2}+Z_{3} w_{s}^{3}+Z_{4} \cos (p \theta-\Omega t) \\
& +Z_{5} w_{s} \cos (p \theta-\Omega t)+Z_{6} w_{s}^{2} \cos (p \theta-\Omega t) \\
& +Z_{7} w_{s}^{3} \cos (p \theta-\Omega t)+\cdots,
\end{aligned}
$$

where

$$
\begin{aligned}
& Z_{0}=\frac{1}{4 \mu_{0}}\left(B_{\delta}^{2}+B_{v}^{2}+B_{\mu}^{2}\right), \quad Z_{1}=-\frac{2 Z_{0}}{\delta_{0}}, \quad Z_{2}=\frac{3 Z_{0}}{\delta_{0}^{2}}, \\
& Z_{3}=-\frac{4 Z_{0}}{\delta_{0}^{3}}, \quad Z_{4}=\frac{1}{2 \mu_{0}} B_{v} B_{\mu}, \quad Z_{5}=-\frac{2 Z_{4}}{\delta_{0}}, \\
& Z_{6}=\frac{3 Z_{4}}{\delta_{0}^{2}}, \quad Z_{7}=-\frac{4 Z_{4}}{\delta_{0}^{3}} .
\end{aligned}
$$

And $\Omega=\omega_{\nu} \pm \omega_{\mu}$ are the frequencies of electromagnetic force corresponding to $p=\nu \pm \mu . B_{\delta}, B_{v}$, and $B_{\mu}$ are fundamental components, the harmonic components of stator windings and rotor windings, respectively. The phase angles are neglected in vibration analysis. Equation (2) shows that there is mutual interaction between the electromagnetic and mechanical behaviors. That is the major problem focused on in this paper.

\section{Double-Shell Stator Model}

Several stator models are presented in previous works for kinds of stator structures, such as single thin circular ring model, two concentric circular rings model, single thick cylindrical shell model [3], and two coaxial cylindrical shells named double-shell system here. A three-phase asynchronous machine is considered in this paper; its stator core and frame are interconnected by key bars. The length of the stator core is shorter than that of the frame. Thus, the doubleshell stator model can be established as in Figure 1.

Based on Hamilton's Principle, the displacement variational principle for nonlinear vibration of thin elastic shell can be expressed as

$$
\begin{aligned}
& \delta \int_{0}^{t}(\mathrm{KE}-\mathrm{PE}) d t \\
& \quad+\int_{0}^{t} \iint_{s}\left(q_{x} \delta u+q_{\theta} \delta v+q_{r} \delta w\right) R d x d \theta d t=0,
\end{aligned}
$$

where $\mathrm{KE}$ is kinetic energy, and PE is potential energy of thin shell. $q_{x}, q_{\theta}$, and $q_{r}$ are components of electromagnetic 


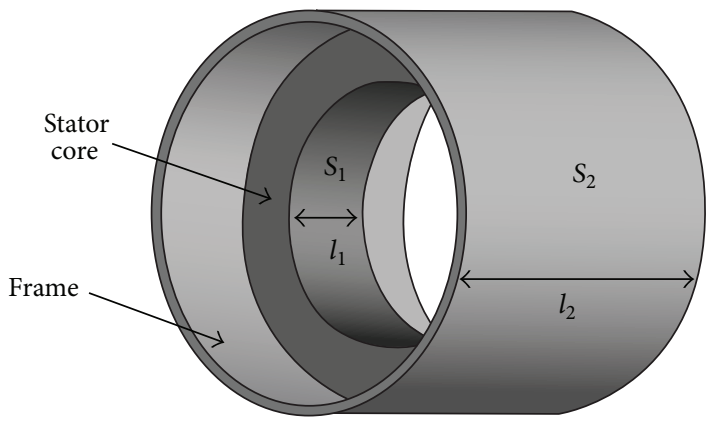

(a)

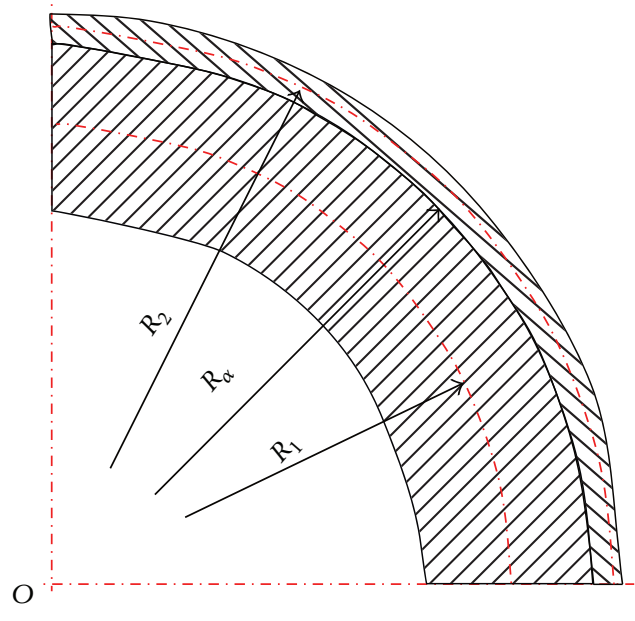

(b)

FIgURE 1: Double-shell stator system.

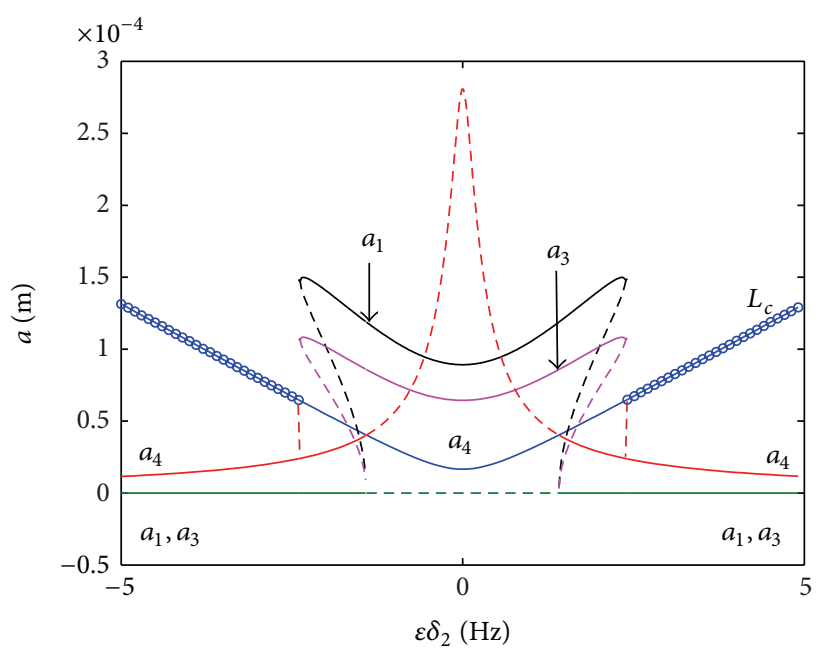

(a)

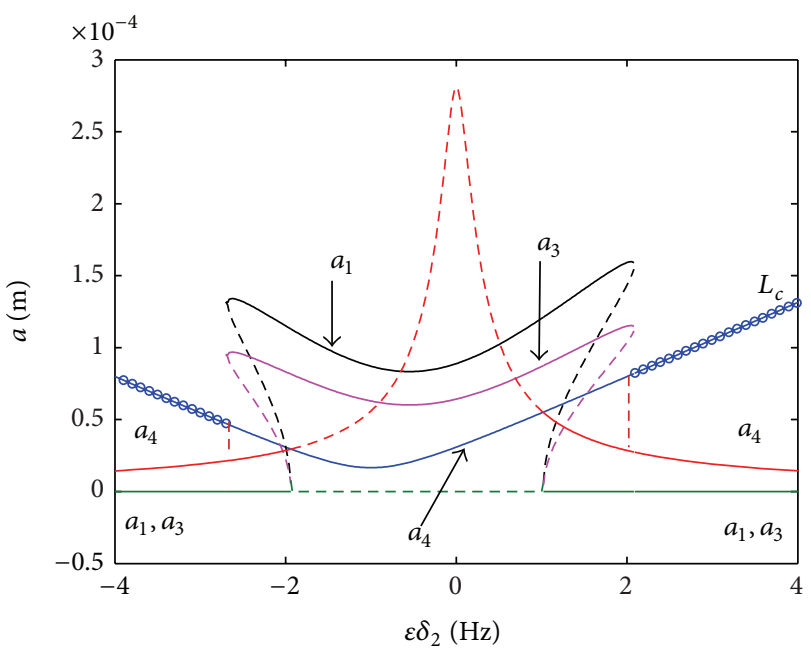

(b)

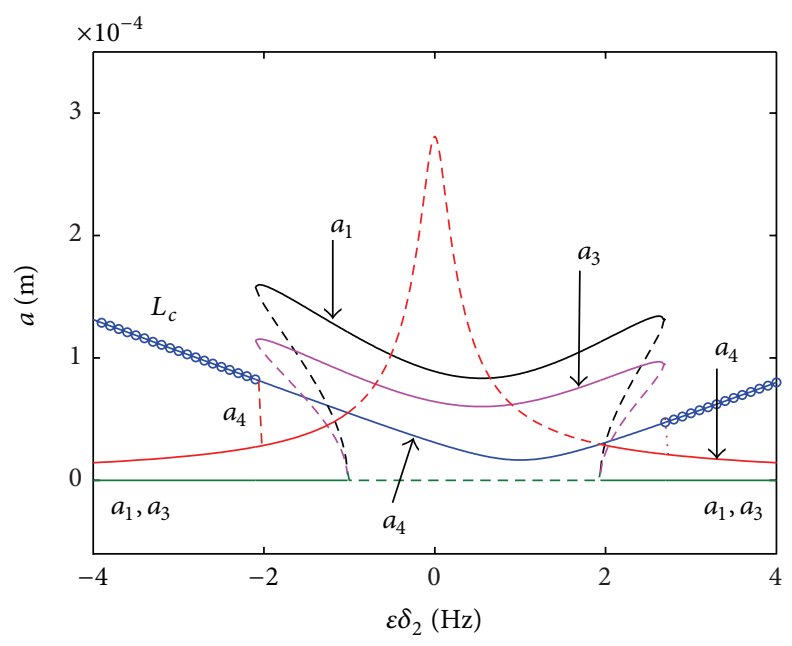

(c)

FIGURE 2: The frequency-response curves. (a) $\delta_{1}=0$, (b) $\delta_{1}>0$, and (c) $\delta_{1}<0$. 


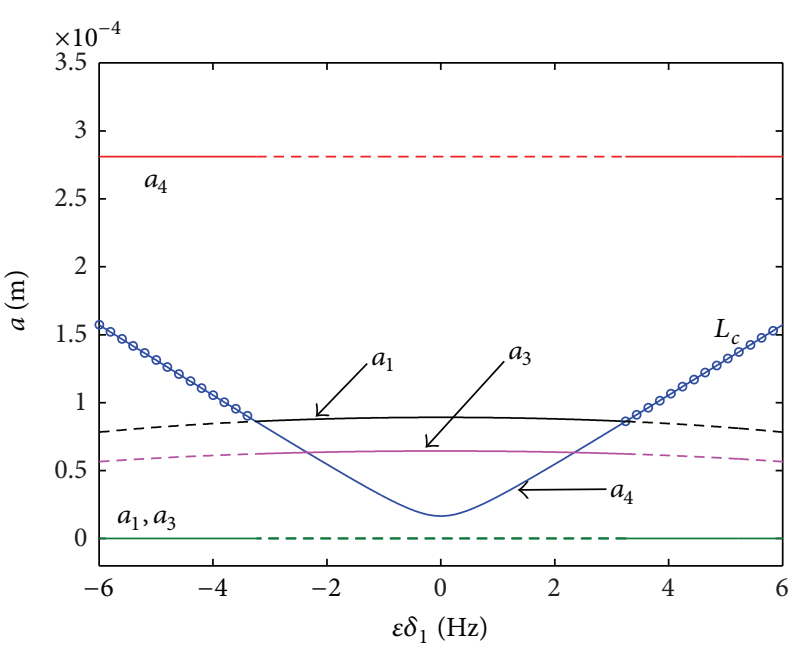

(a)

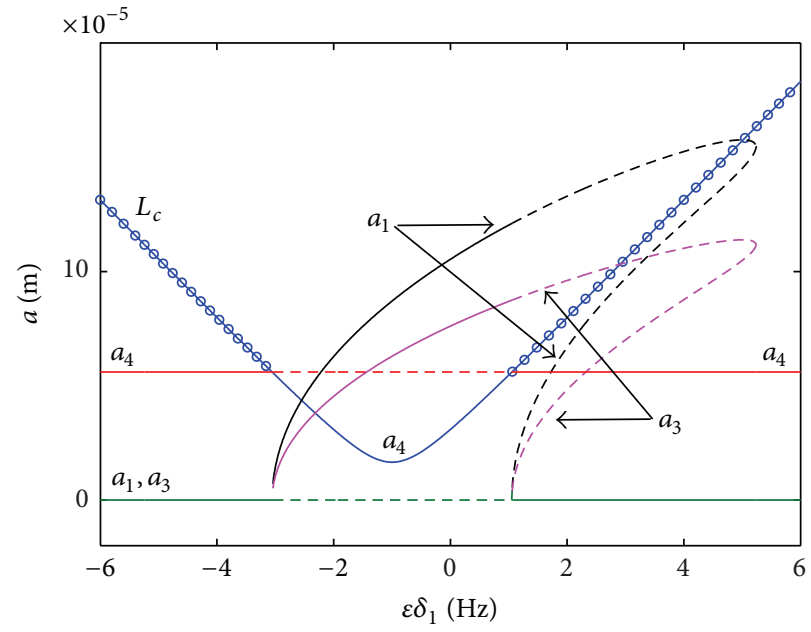

(b)

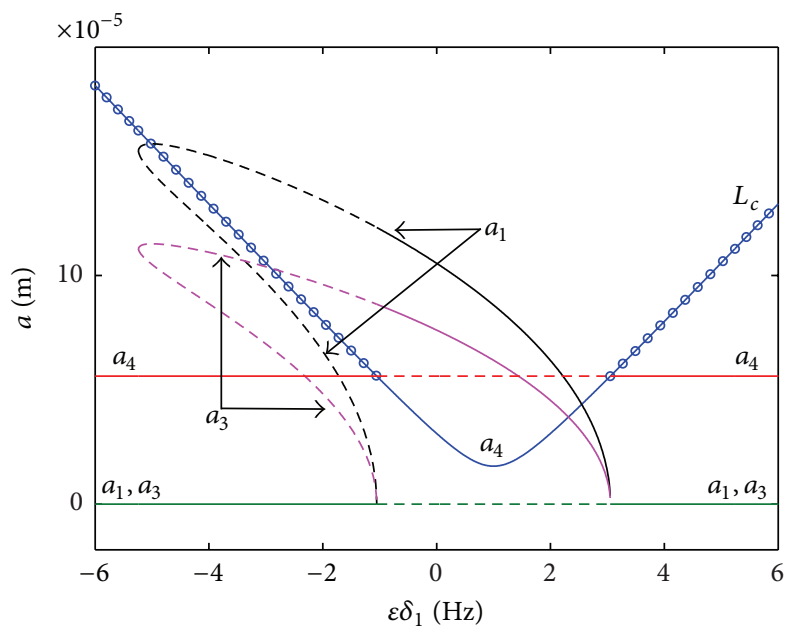

(c)

Figure 3: The frequency-response curves. (a) $\delta_{2}=0$, (b) $\delta_{2}>0$, and (c) $\delta_{2}<0$.

force, and $x, \theta$, and $r$ are cylindrical coordinates, respectively. The coincident part of the double-shell stator system can be considered as being fixed together by a supposed force $P(x, \theta) \sin \omega_{i} t$. Then, the force conditions of the stator core and the frame can be written as

Stator core: $q_{r}^{s}(x, \theta, t)=-\frac{R_{\alpha}}{R_{1}} P(x, \theta) \sin \omega_{i} t+\frac{R_{\beta}}{R_{1}} q_{r}(x, \theta, t)$,

Frame: $q_{r}^{r}(x, \theta, t)=\frac{R_{\alpha}}{R_{1}} P(x, \theta) \sin \omega_{i} t$

where $R_{\beta}$ is the centroid radius of the stator core. The superscript $s$ is for the stator core and $r$ is for the frame. The displacement solutions can be written as the products of periodic function $T_{i}(t)$ and modal shape functions $U_{i}, V_{i}, W_{i}$ determined by the boundary condition as

$$
\begin{gathered}
u(x, \theta, t)=\sum_{i} T_{i}(t) U_{i}(x, \theta), \\
v(x, \theta, t)=\sum_{i} T_{i}(t) V_{i}(x, \theta), \\
w(x, \theta, t)=\sum_{i} T_{i}(t) W_{i}(x, \theta),
\end{gathered}
$$

where $i$ is orders of mode shapes. Equation (6) can be used to determine the displacement components of the stator core and the frame, respectively. Considering the orthogonality of mode shapes and the displacement boundary condition of 


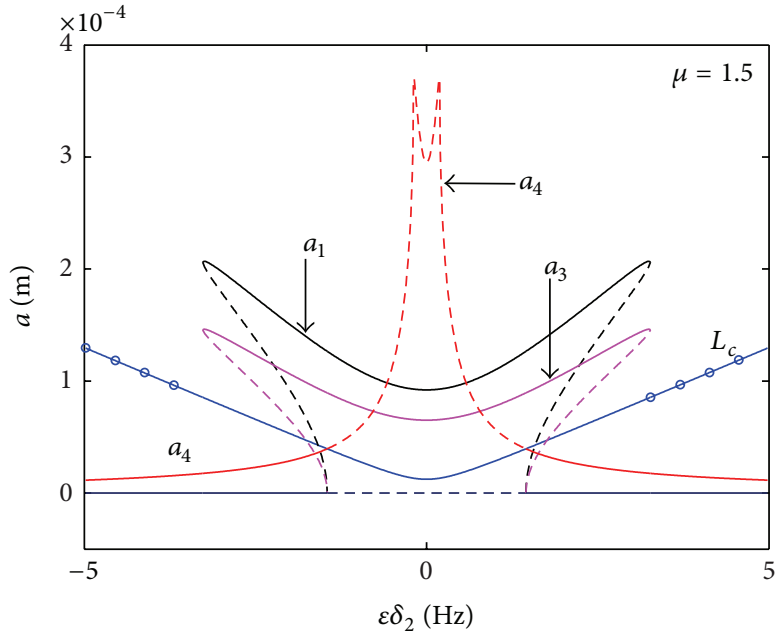

(a)

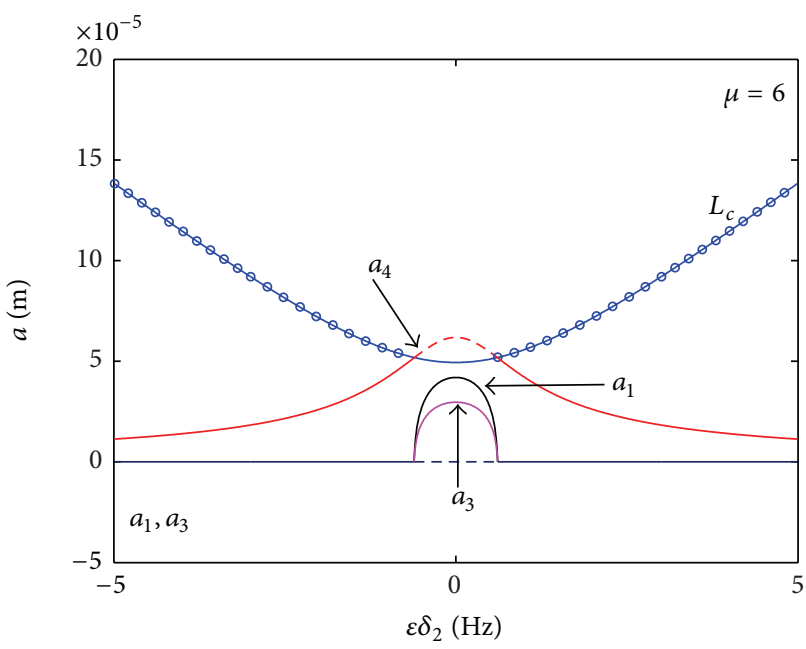

(c)

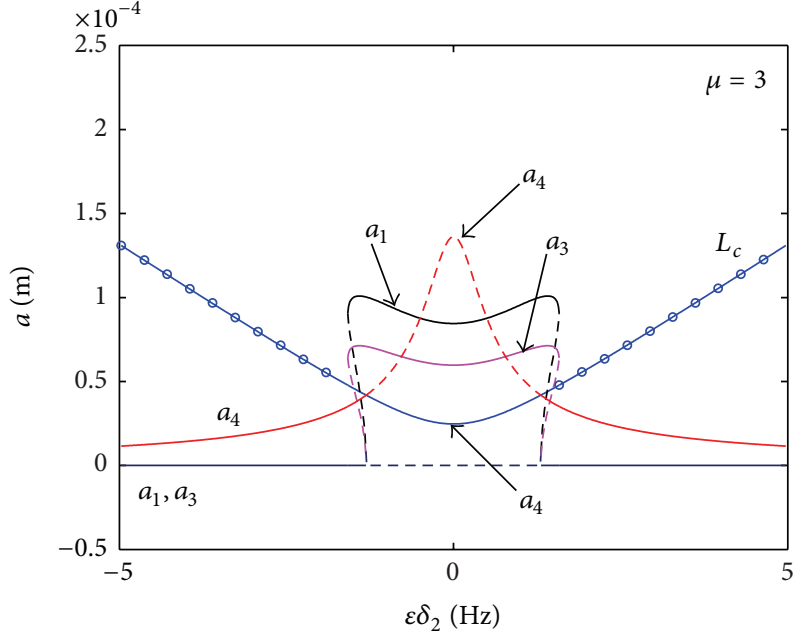

(b)

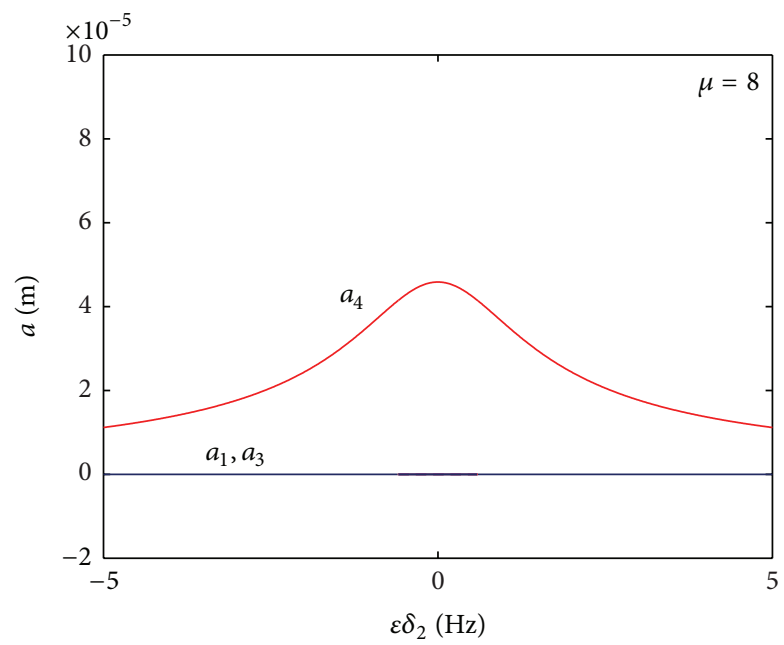

(d)

FIgURE 4: The frequency-response curves.

$w_{s} \equiv w_{r}$ in the coincident region $s_{1}$, the vibration equation with modal damping can be obtained as

$$
\begin{aligned}
\ddot{T}_{i}(t) & +2 \mu_{i} \dot{T}_{i}(t)+\omega_{i}^{2} T_{i}(t) \\
& =\frac{R_{\beta}}{M_{i s}+M_{i r}} \iint_{s_{1}} q_{r}(x, \theta, t) W_{i s} d x d \theta,
\end{aligned}
$$

where $\mu_{i}$ is modal damping coefficient, and $\omega_{i}$ is the natural frequency of double-shell system.

\section{Nonlinear Dynamic Equations}

Substituting (2) into (7), the nonlinear dynamic equations of the stator system with the interaction between the radial displacement of the stator core and the air-gap magnetic field are obtained as

$$
\begin{aligned}
\ddot{T}_{i}(t) & +2 \mu_{i} \dot{T}_{i}(t)+\bar{\omega}_{i}^{2} T_{i}(t) \\
= & \eta_{i 1} \sum_{j} \sum_{k} \Gamma_{i j k} T_{j}(t) T_{k}(t) \\
& +\eta_{i 4} \sum_{j} \sum_{k} \Gamma_{i j k} T_{j}(t) T_{k}(t) \cos \Omega t+\cdots \quad(i \neq p), \\
\ddot{T}_{p}(t) & +2 \mu_{p} \dot{T}_{p}(t)+\bar{\omega}_{p}^{2} T_{p}(t) \\
= & \eta_{p 1} \sum_{j} \sum_{k} \Gamma_{p j k} T_{j}(t) T_{k}(t) \\
& +\eta_{p 4} \sum_{j} \sum_{k} \Gamma_{p p j k} T_{j}(t) T_{k}(t) \cos \Omega t+2 F_{p} \cos \Omega t+\cdots
\end{aligned}
$$

$$
(i=p)
$$




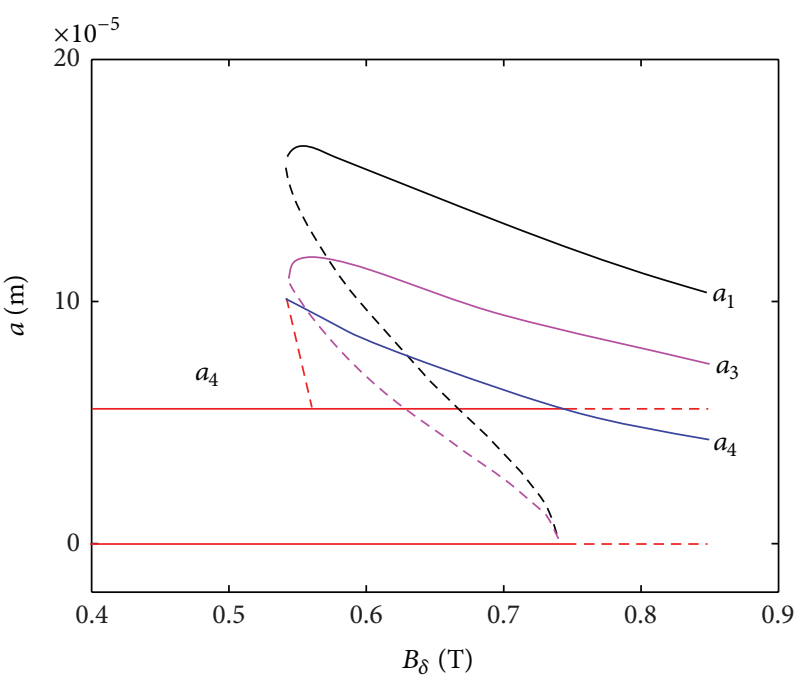

(a)

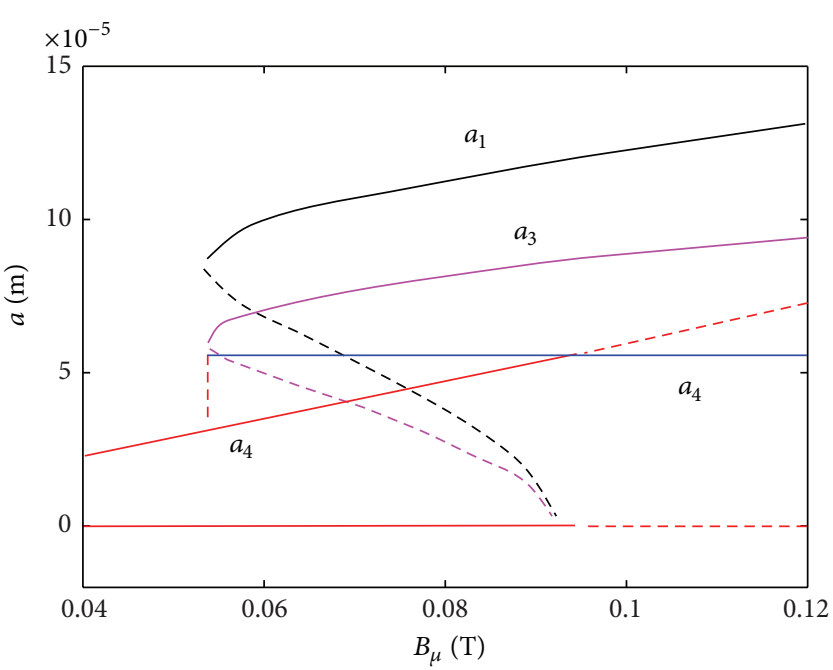

(b)

FIgURE 5: The amplitude-magnetic flux-density response curves.

where $j, k$ are orders of mode shapes distinguishing from $i$, and

$$
\bar{\omega}_{i}^{2}=\omega_{i}^{2}-\frac{R_{\beta}}{M_{i s}+M_{i r}} \iint_{s_{1}} Z_{1} w_{s} W_{i s} d x d \theta
$$

are the frequencies of the stator system in working state. $\Gamma_{i j k}$ are the coupling coefficients which present the interaction of different mode shapes. And $\eta_{i n}$ and $\Gamma_{i j k}$ are expressed as

$$
\begin{gathered}
\eta_{(i, p), 1}=\frac{1}{\delta_{0}^{2}} \frac{3 Z_{0} l_{1} R_{\beta}}{M_{(i, p), s}+M_{(i, p), r}}, \\
\eta_{(i, p), 4}=\frac{1}{\delta_{0}^{2}} \frac{3 Z_{4} l_{1} R_{\beta}}{M_{(i, p), s}+M_{(i, p), r}}, \\
2 F_{p}=\frac{\pi Z_{4} l_{1} R_{\beta}}{M_{p s}+M_{p r}}, \\
\Gamma_{i j k}=\int_{0}^{2 \pi} \cos i \theta \cdot \cos j \theta \cdot \cos k \theta d \theta .
\end{gathered}
$$

Equations (8a) and (8b) show that the forced excitation term exists in the dynamic equations if and only if $i=p$. Therefore, both of the two equations are necessary for the combined internal and principal parametric resonances. All the other nonlinear terms are not important for the steadystate analysis and they will decay with time.

The relationship of internal resonance can be expressed by introducing detuning parameter $\delta_{1}$, and the nearness of excitation frequency $\Omega$ to one natural frequency $\bar{\omega}_{p}$ can be expressed by introducing the external detuning parameter $\delta_{2}$ according to

$$
\bar{\omega}_{4}=\bar{\omega}_{1}+\bar{\omega}_{3}+\varepsilon \delta_{1}, \quad \Omega=\bar{\omega}_{4}+\varepsilon \delta_{2} .
$$

We introduce small parameter $\varepsilon \ll 1$ to transform the scale $\mu_{i} \rightarrow \varepsilon \mu_{i}^{\prime}$ and $\eta_{i n} \rightarrow \varepsilon \eta_{i n}^{\prime}$, and the method of multiple scales [16] is applied to solve (8a) and (8b) as

$$
T_{i}(t, \varepsilon)=T_{i 0}\left(T_{0}, T_{1}\right)+\varepsilon T_{i 1}\left(T_{0}, T_{1}\right) \quad(i=1,3,4),
$$

where $T_{0}=t$ is a fast time scale and $T_{1}=\varepsilon t$ is a slow time scale. Inserting (12) into (8a) and (8b), and separating at each order of $\varepsilon$, one obtains

$$
\begin{aligned}
& O\left(\varepsilon^{0}\right): D_{0}^{2} T_{i 0}+\bar{\omega}_{i}^{2} T_{i 0}=0, \\
& O\left(\varepsilon^{1}\right): D_{0}^{2} T_{i 1}+\bar{\omega}_{i}^{2} T_{i 1} \\
& =-2 D_{0} D_{1} T_{i 0}-2 \mu_{i}^{\prime} D_{0} T_{i 0}+\eta_{i 1}^{\prime} \sum_{j} \sum_{k} \Gamma_{i j k} T_{j 0} T_{k 0} \\
& \quad+\eta_{i 4}^{\prime} \sum_{j} \sum_{k} \Gamma_{p i j k} T_{j 0} T_{k 0} \cos \Omega T_{0}+\cdots \quad(i \neq p), \\
& O\left(\varepsilon^{0}\right): D_{0}^{2} T_{p 0}+\bar{\omega}_{p}^{2} T_{p 0}=0, \\
& O\left(\varepsilon^{1}\right): D_{0}^{2} T_{p 1}+\bar{\omega}_{p}^{2} T_{p 1} \\
& =-2 D_{0} D_{1} T_{p 0}-2 \mu_{p}^{\prime} D_{0} T_{p 0} \\
& \quad+\eta_{p 1}^{\prime} \sum_{j} \sum_{k} \Gamma_{p j k} T_{j 0} T_{k 0} \\
& \quad+\eta_{p 4}^{\prime} \sum_{j} \sum_{k} \Gamma_{p p j k} T_{j 0} T_{k 0} \cos \Omega T_{0}+2 F_{p}^{\prime} \cos \Omega T_{0}+\cdots
\end{aligned}
$$




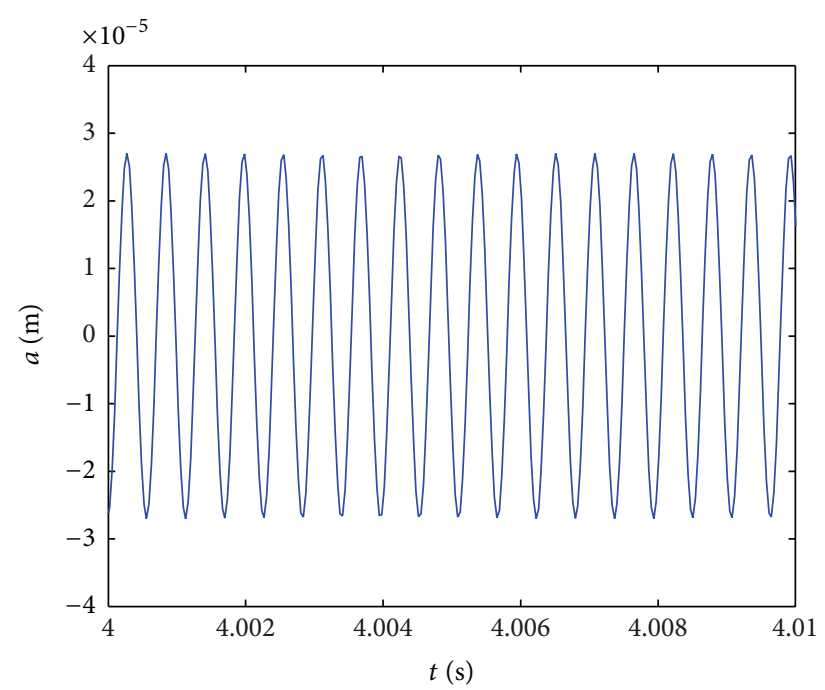

(a) Time history

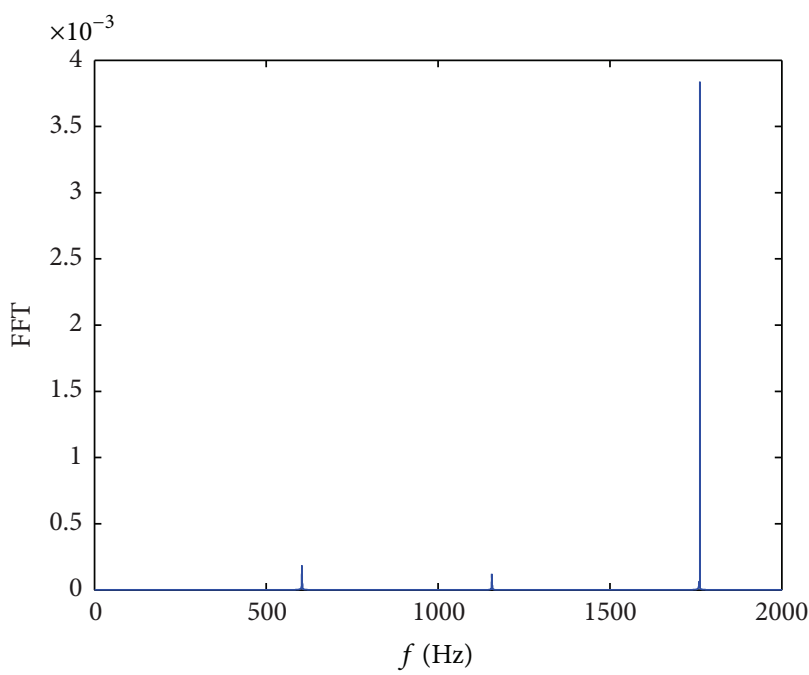

(c) FFT

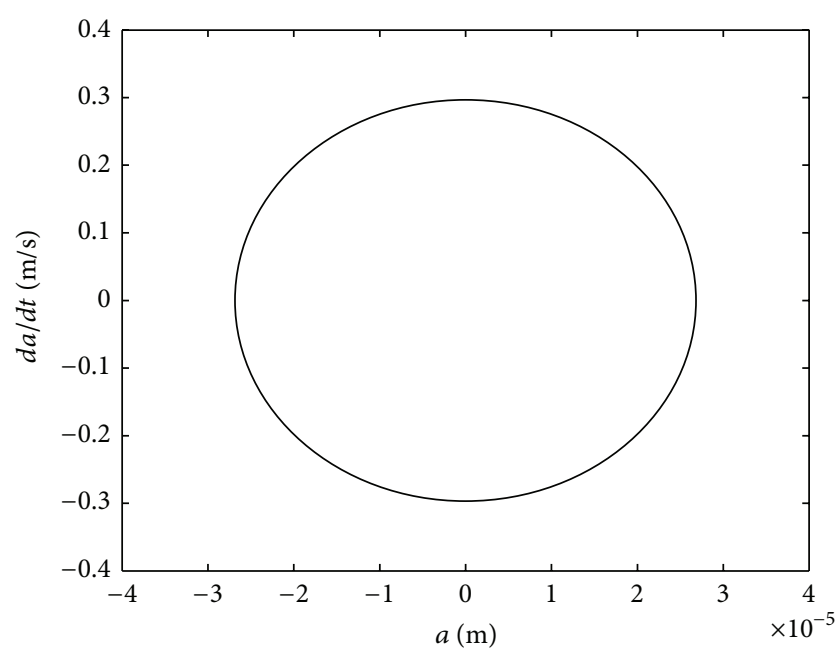

(b) Phase chart

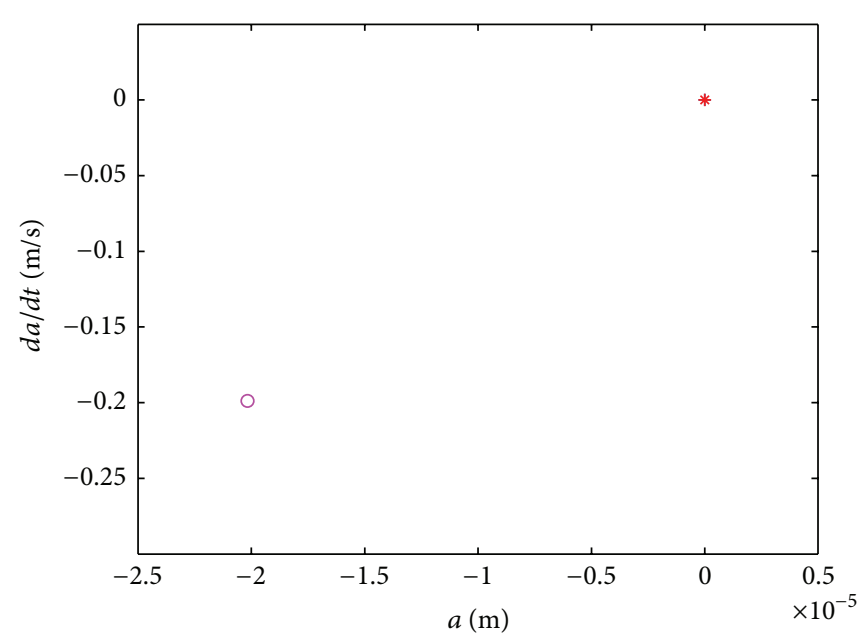

(d) Poincaré section

Figure 6: Dynamic characteristic with $\varepsilon \delta_{1}=0.12, \varepsilon \delta_{2}=0.48, \mu=8, B_{\delta}=0.75 \mathrm{~T}$, and $B_{\mu}=0.1 \mathrm{~T}$.

where $D_{n}=\partial / \partial T_{n}$. At $O\left(\varepsilon^{0}\right)$ the solution can be written in the following convenient form:

$$
T_{i 0}\left(T_{0}, T_{1}\right)=\frac{a_{i}\left(T_{1}\right)}{2} \exp \left[j\left(\omega_{i} T_{0}+\phi_{i}\left(T_{1}\right)\right)\right]+\text { c.c., }
$$

where $a_{i}$ and $\phi_{i}$ are real. $a_{i}$ are the amplitudes; $\phi_{i}$ are the phases. c.c. stands for complex conjugate and $j^{2}=-1$.

Substituting (14) into the $\varepsilon^{1}$-order equations in (13a) and (13b) leads to the differential equations, which can be written as a six-dimensional dynamical system by separating real and imaginary parts as

$$
\begin{gathered}
a_{1}^{\prime}=\mu_{1} a_{1}-\frac{\eta_{11}}{4 \bar{\omega}_{1}} \Gamma_{143} a_{3} a_{4} \sin \gamma_{1}, \\
a_{1} \phi_{1}^{\prime}=-\frac{\eta_{11}}{4 \bar{\omega}_{1}} \Gamma_{143} a_{3} a_{4} \cos \gamma_{1},
\end{gathered}
$$




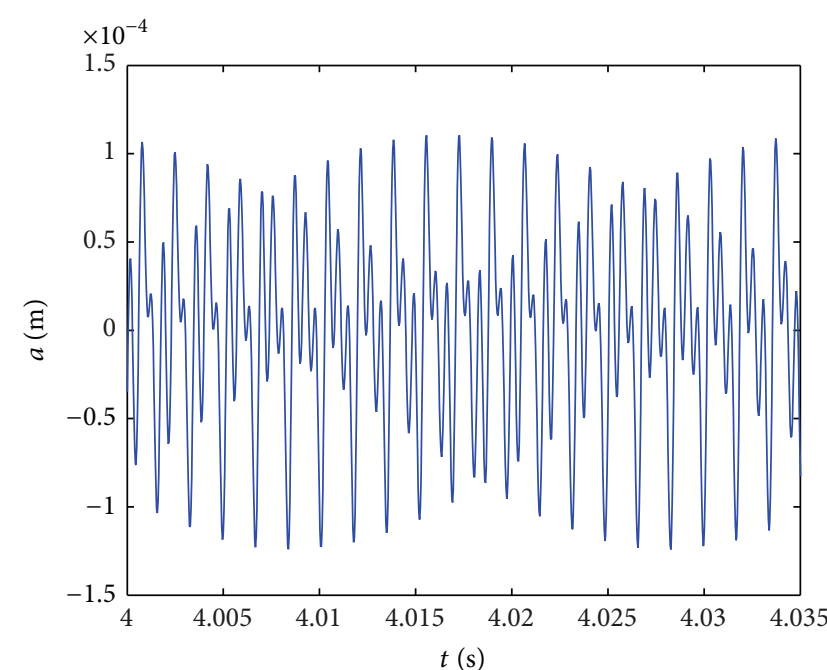

(a) Time history

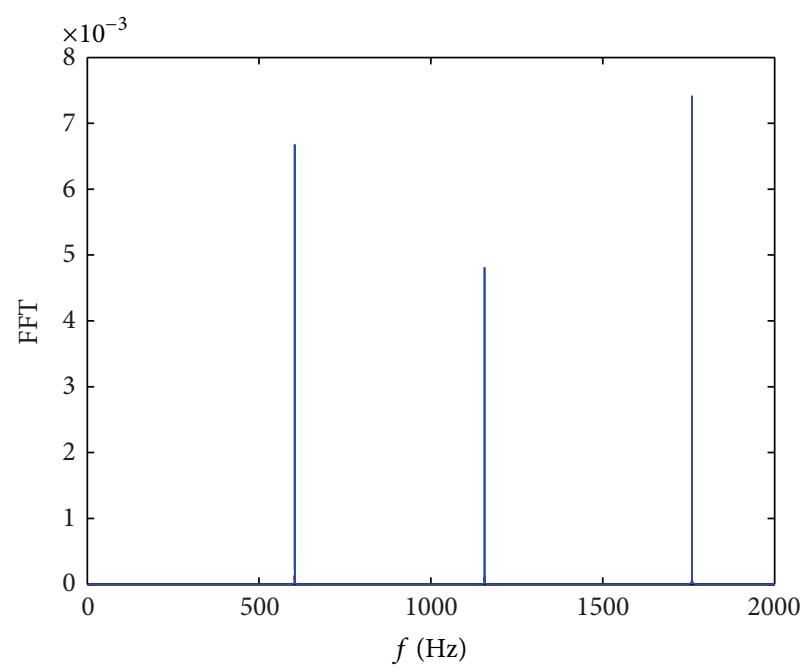

(c) FFT

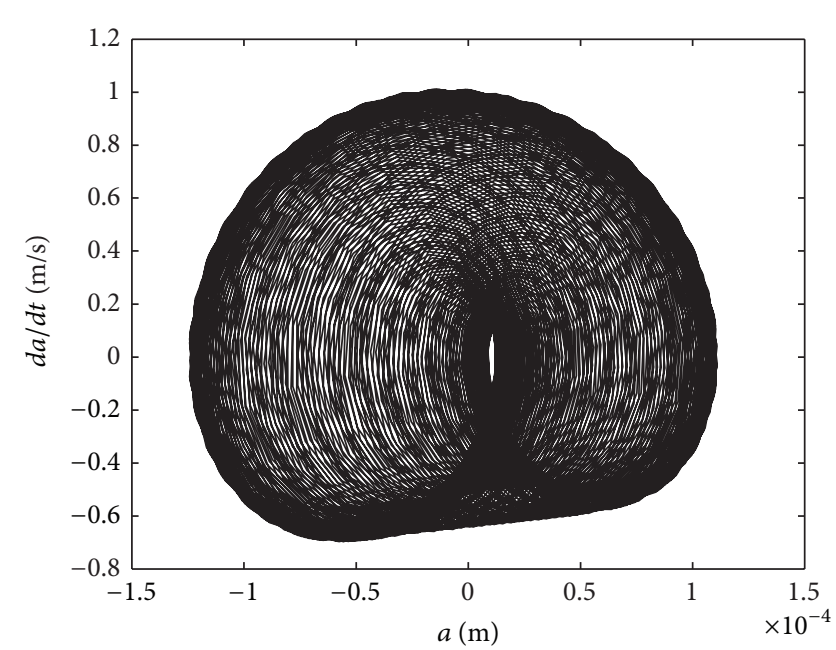

(b) Phase chart

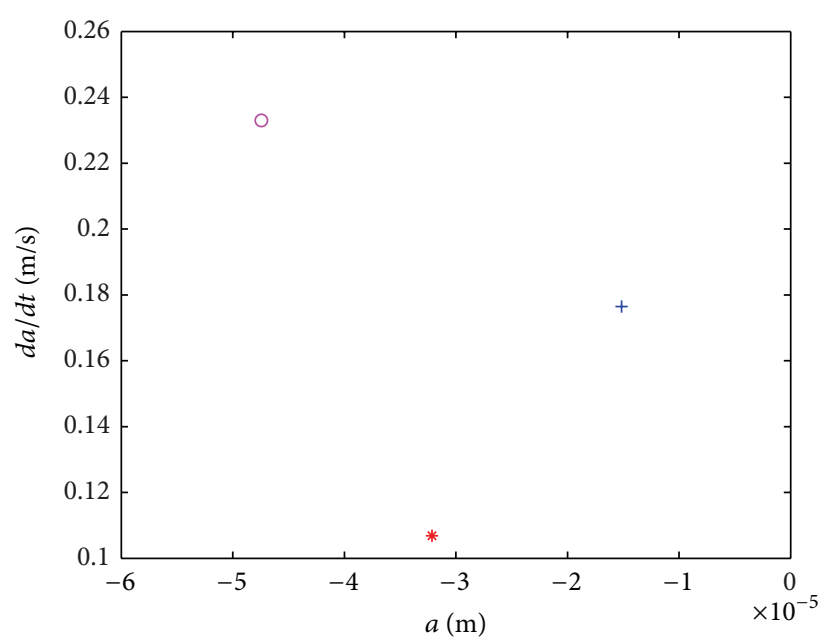

(d) Poincaré section

FIgURE 7: Dynamic characteristic with $\varepsilon \delta_{1}=0.12, \varepsilon \delta_{2}=0.48, \mu=6, B_{\delta}=0.75 \mathrm{~T}$, and $B_{\mu}=0.1 \mathrm{~T}$.

where $(\cdot)^{\prime}$ stands for the derivation with respect to $T_{1}, \gamma_{1}=$ $\delta_{1} T_{1}-\phi_{1}-\phi_{3}+\phi_{4}$, and $\gamma_{2}=\delta_{2} T_{1}-\phi_{4}$.

For the steady-state solution, by canceling the time derivatives $\left(a_{1}^{\prime}=a_{3}^{\prime}=a_{4}^{\prime}=\gamma_{1}^{\prime}=\gamma_{2}^{\prime}=0\right)$, one can obtain that only two kinds of solutions are possible. In the absence of the internal resonance, the forced excitation can excite only one mode at a time. In this case, the amplitude of the stator system can be only expressed by the fourth mode as

$$
\begin{gathered}
a_{1}=0, \quad a_{3}=0, \\
\Lambda_{44}^{2} a_{4}^{4}+\left[2 \Lambda \Lambda_{44}-\mu_{4}^{2}-\left(\varepsilon \delta_{2}\right)^{2}\right] a_{4}^{2}+\Lambda^{2}=0 .
\end{gathered}
$$

But in the presence of the internal resonance, the combined internal and principal parametric resonances occur, and the amplitude of the stator system can be obtained as

$$
\begin{aligned}
& a_{1}^{2}=\frac{1}{\Lambda_{3} \Lambda_{4}} \sqrt{\frac{\mu_{3}}{\mu_{1}}}\left[P_{1} \pm \sqrt{P_{2}^{2}-P_{3}^{2}}\right], \quad a_{3}^{2}=\frac{\mu_{1} \Lambda_{3}}{\mu_{3} \Lambda_{1}} a_{1}^{2}, \\
& a_{4}^{2}=\frac{\mu_{1} \mu_{3}}{\Lambda_{1} \Lambda_{3}}\left[1+\frac{\left(\varepsilon \delta_{1}+\varepsilon \delta_{2}\right)^{2}}{\left(\mu_{1}+\mu_{3}\right)^{2}}\right],
\end{aligned}
$$

where

$$
\begin{aligned}
& P_{1}=\frac{\sqrt{\mu_{1} \mu_{3}}}{\mu_{1}+\mu_{3}}\left[\varepsilon \delta_{2}\left(\varepsilon \delta_{1}+\varepsilon \delta_{2}\right)-\mu_{4}\left(\mu_{1}+\mu_{3}\right)\right], \\
& P_{2}=\sqrt{\Lambda_{1} \Lambda_{3}}\left(\Lambda+\Lambda_{44} a_{4}^{2}\right),
\end{aligned}
$$




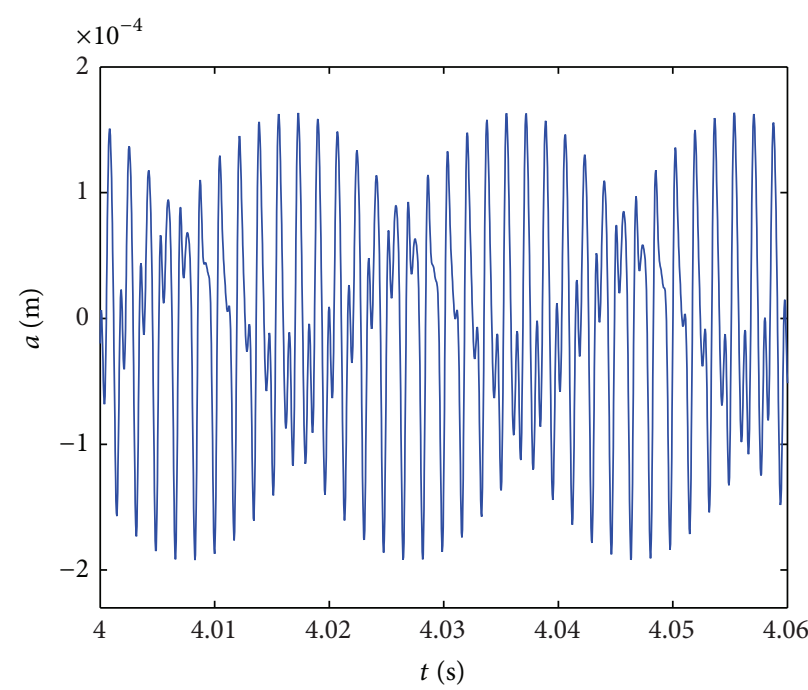

(a) Time history

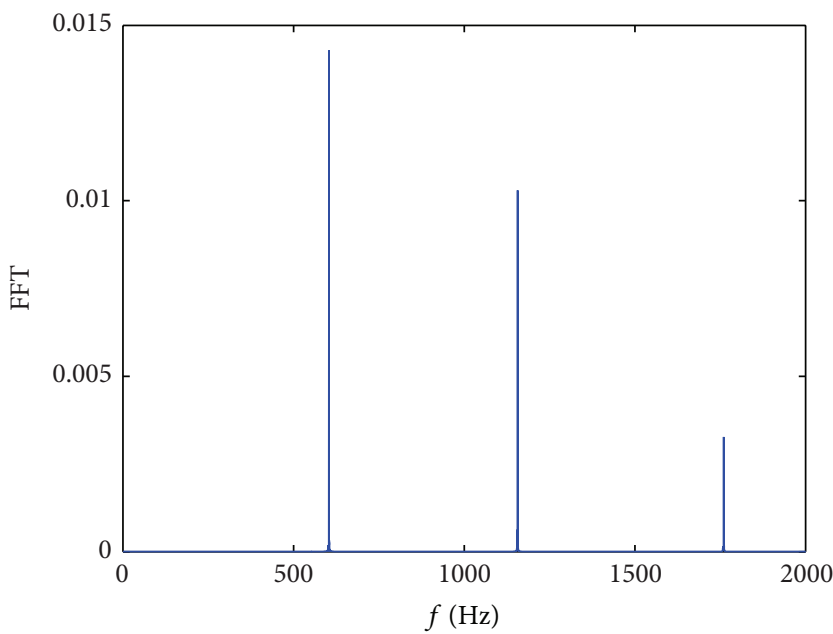

(c) FFT

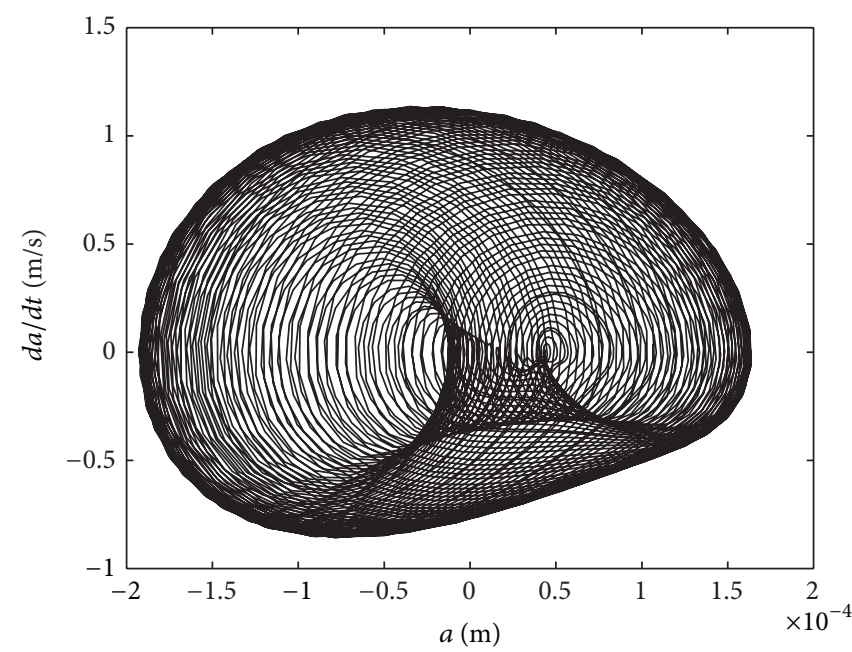

(b) Phase chart

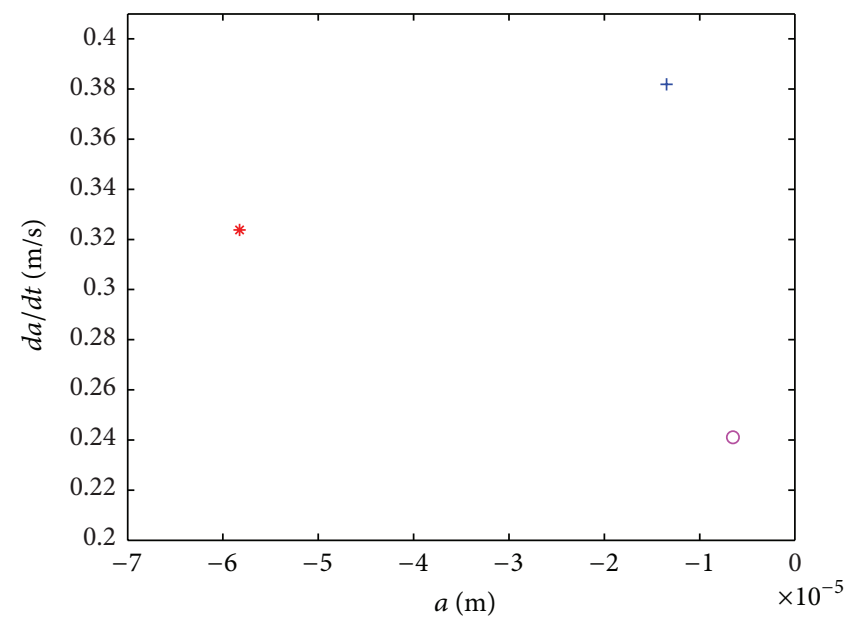

(d) Poincaré section

FIgURE 8: Dynamic characteristic with $\varepsilon \delta_{1}=0.12, \varepsilon \delta_{2}=0.48, \mu=2, B_{\delta}=0.75 \mathrm{~T}$, and $B_{\mu}=0.1 \mathrm{~T}$.

$P_{3}=\frac{\sqrt{\mu_{1} \mu_{3}}}{\mu_{1}+\mu_{3}}\left[\varepsilon \delta_{2}\left(\mu_{1}+\mu_{3}\right)+\mu_{4}\left(\varepsilon \delta_{1}+\varepsilon \delta_{2}\right)\right]$,

$$
\begin{aligned}
& \Lambda_{1}=\frac{\eta_{11}}{4 \bar{\omega}_{1}} \Gamma_{143}, \quad \Lambda_{3}=\frac{\eta_{31}}{4 \bar{\omega}_{3}} \Gamma_{341}, \quad \Lambda_{4}=\frac{\eta_{41}}{4 \bar{\omega}_{4}} \Gamma_{413} \\
& \Lambda_{44}=\frac{\eta_{44}}{4 \bar{\omega}_{4}} \Gamma_{4444}, \quad \Lambda=\frac{F_{4}}{\bar{\omega}_{4}}
\end{aligned}
$$

The stability analysis can be derived by computing the Jacobian matrix of (15) and the existence condition of real solutions. If all the real parts of the eigenvalues from the Jacobian matrix are negative, the corresponding motion is stable; otherwise it is unstable. Finally, the following stability condition of the continuous system is obtained as

$$
\begin{aligned}
& L_{a}=P_{1} \pm \sqrt{P_{2}^{2}-P_{3}^{2}}>0, \quad L_{b}=P_{2}^{2}-P_{3}^{2} \geq 0 \\
& L_{c}=\left\{\frac{\mu_{1} \mu_{3}}{\Lambda_{1} \Lambda_{3}}\left[1+\frac{\left(\varepsilon \delta_{1}+\varepsilon \delta_{2}\right)^{2}}{\left(\mu_{1}+\mu_{3}\right)^{2}}\right]\right\}^{1 / 2} \geq a_{4} .
\end{aligned}
$$

\section{Numerical Simulation and Discussion}

In this section, the fourth-order Runge-Kutta method is used to solve (15) for more insight into the combined internal and principal parametric resonances by assuming $\mu_{1}=\mu_{3}=\mu_{4}=$ $\mu$. The stationaryfrequency-response curves are illustrated 


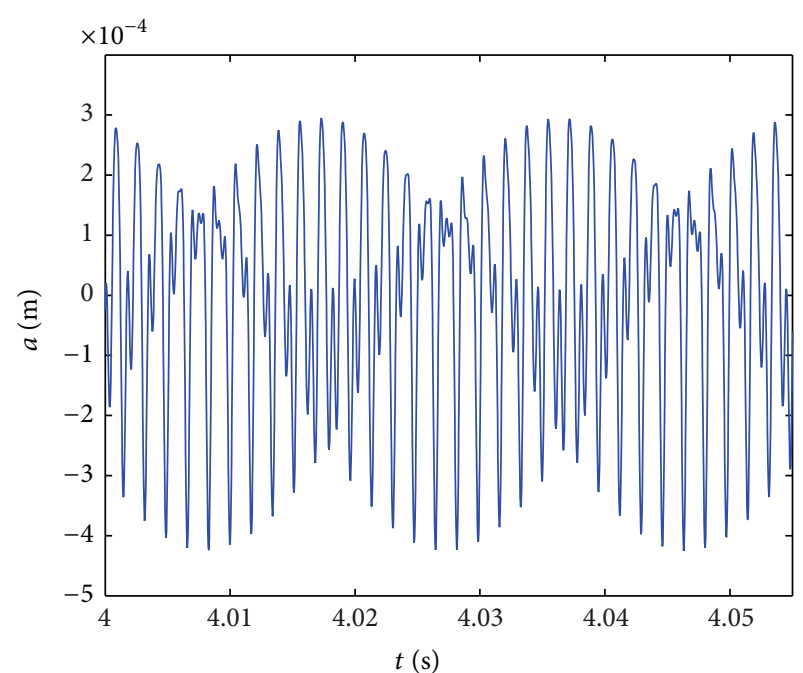

(a) Time history

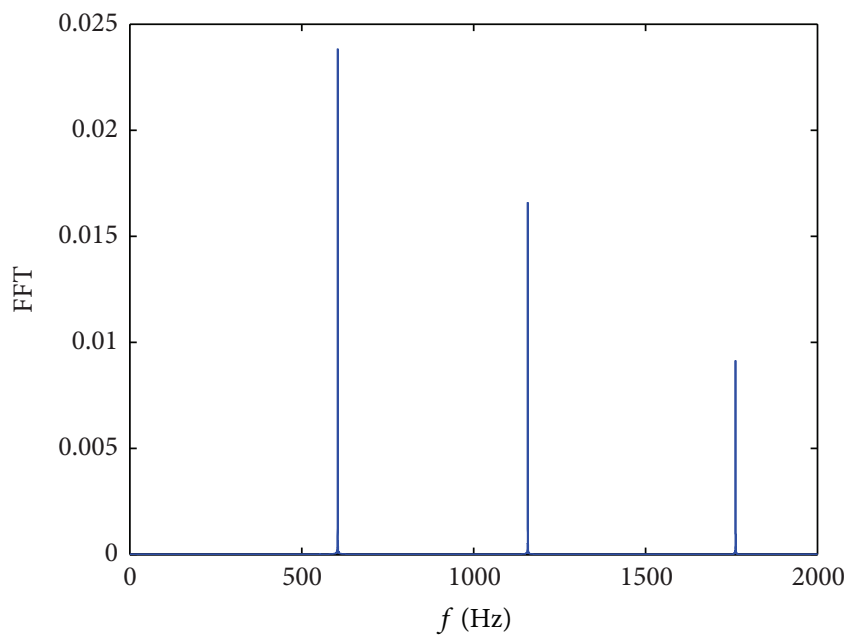

(c) FFT

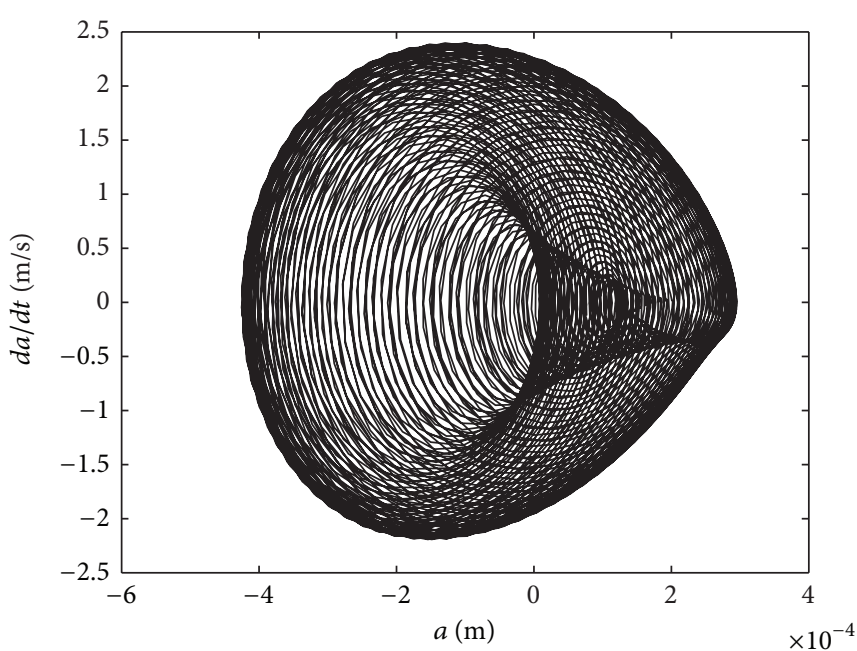

(b) Phase chart

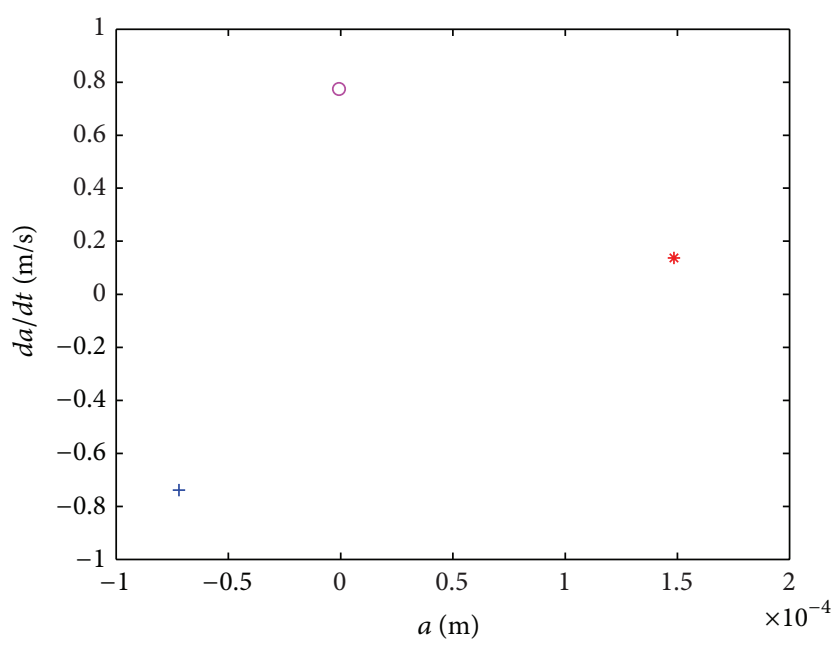

(d) Poincaré section

FIgURE 9: Dynamic characteristic with $\varepsilon \delta_{1}=0.12, \varepsilon \delta_{2}=0.48, \mu=2, B_{\delta}=0.5 \mathrm{~T}$, and $B_{\mu}=0.2 \mathrm{~T}$.

in Figures 2-4. In these figures, the ordinate $a$ represents the steady-state amplitude and the abscissa $\varepsilon \delta$ denotes the frequency detuning parameter. Solid and broken lines in these figures represent the stable and unstable solutions, respectively.

5.1. Influence of Detuning Parameters. Figure 2 shows the stationary frequency-response curves with three cases of internal resonance detuning parameter $\delta_{1}$. All the modal damping coefficients are assumed to be 2 . When the internal resonance detuning parameter $\delta_{1}$ is zero, the response curves are symmetric and present M-shape. If $\delta_{1}>0$, the symmetry axis moves to the left based on (17) and inclines towards right. And if $\delta_{1}<0$, the symmetry axis moves to the right and inclines towards left. This means the fourth mode is forcedly and parametrically excited, and the presences of the first and third modes are possible only by transferring energy from the fourth mode to them through internal mechanism. The peak value of $a_{4}$, which originates from the combined forced and parametric resonance, is independent of the internal resonance detuning parameter $\delta_{1}$. Once the amplitude $a_{4}$ is larger than $L_{c}$, the solution becomes unstable. The internal resonance detuning parameter $\delta_{1}$ has a direct influence on the energy transfer when the simultaneous resonances are excited.

Figure 3 shows the stationary frequency-response curves with three cases of forced resonance detuning parameter $\delta_{2}$. Similarly, only when $\delta_{2}=0$, the response curves are symmetric. When $a_{1}=a_{3}=0$, there is only the fourth mode of the stator system left under parametrically forced resonance with high amplitude. If $\delta_{2}>0$, the right of $a_{1}$ and $a_{3}$ curves rises, and all the curves move towards left based on (17). On the contrary, if $\delta_{2}<0$, the left of $a_{1}$ and $a_{3}$ curves rises, and all the curves move towards right. This means that the amplitudes of $a_{1}$ and $a_{3}$ increase since the simultaneous 


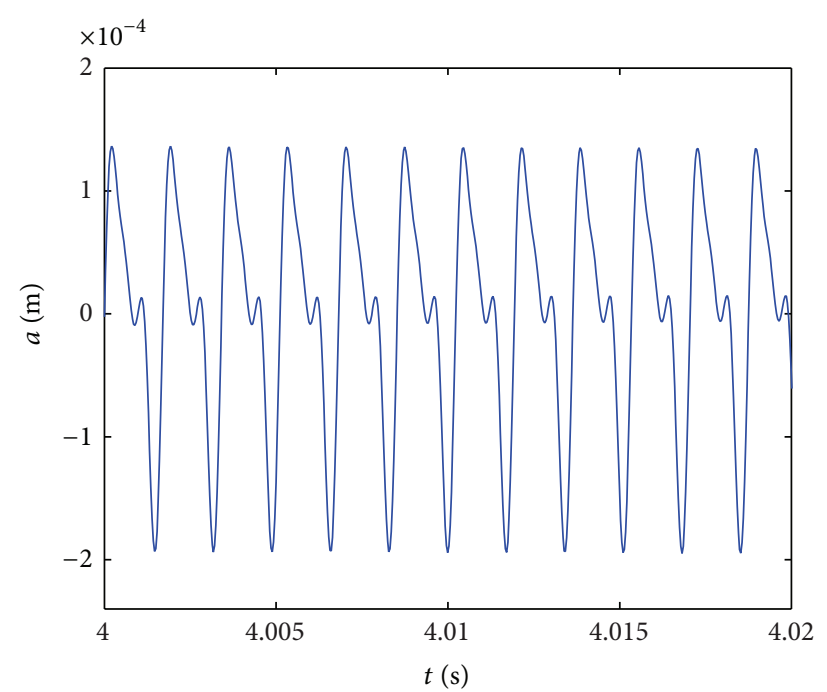

(a) Time history

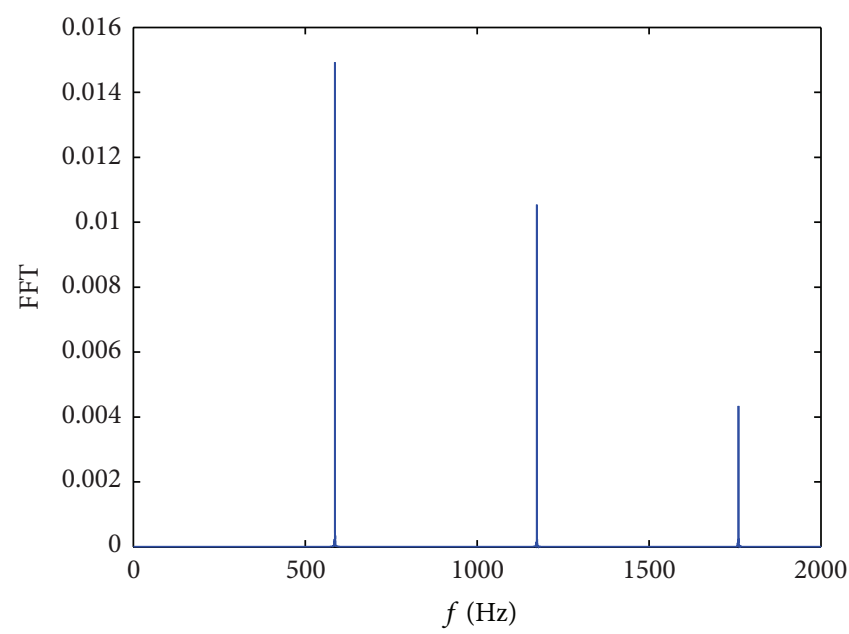

(c) FFT

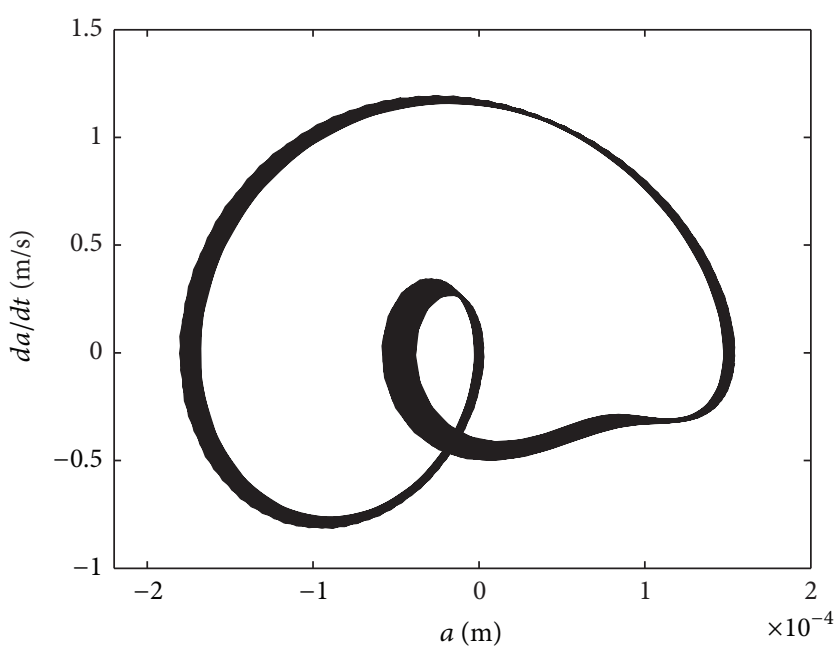

(b) Phase chart

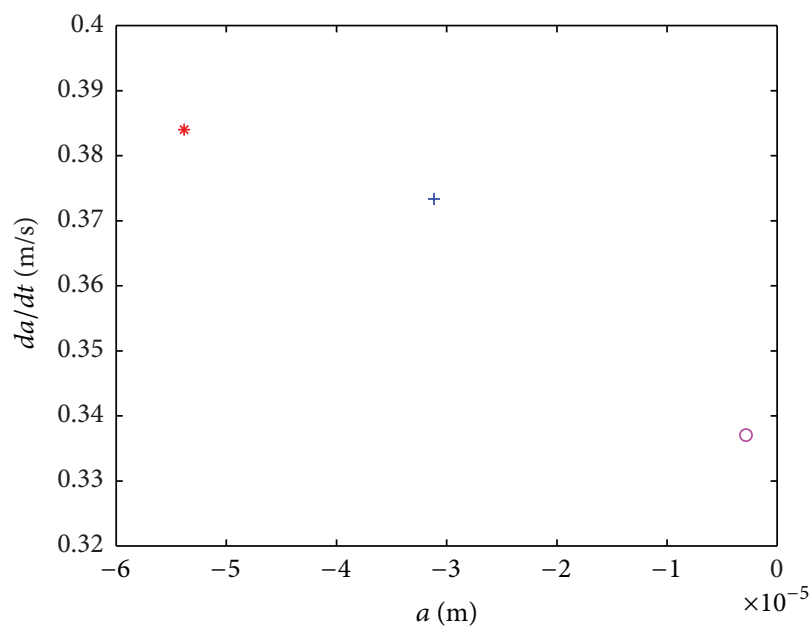

(d) Poincaré section

FIGURE 10: Dynamic characteristic with $\varepsilon \delta_{1}=0.52, \varepsilon \delta_{2}=0.48$, and $\bar{\omega}_{4} \approx 3 \bar{\omega}_{1}$.

resonances occur and then drop to zero when the energy transfer vanishes in Figure 3(b). But the amplitudes of $a_{1}$ and $a_{3}$ jump to a certain value when simultaneous resonances occur and then decrease to zero when the energy transfer vanishes in Figure 3(c).

5.2. Influence of Damping Coefficients. Take the frequencyresponse curves of forced resonance detuning parameter as an example to discuss the influence of damping coefficients. Both the resonance region and the amplitudes of three modes are seen reduced due to the increasing of the damping coefficients in Figure 4. If $\mu<2, a_{4}$ presents an unstable doublepeak curve. And the fourth mode is on combined forced and parametric resonances. When the combined internal and principal parametric resonances are excited, the stable resonance amplitude of $a_{4}$ increases slowly, the unstable one decreases, and the resonance amplitudes of $a_{1}$ and $a_{3}$ reduce gradually with the increase of damping coefficients. When the damping coefficients increase to 6 , the resonance amplitude of $a_{4}$ has been larger than the other modes. Until the damping coefficients increase to 8 , there is only the fourth mode left for the parametrically forced resonance with the absence of the first and third modes. The reason of this phenomenon is that the energy transferred from the fourth mode to the other modes is reducing with the damping coefficients increasing.

5.3. Influence of Electromagnetic Parameters. Figure 5 illustrates the relationship between the amplitude and the magnetic flux-density. The simultaneous resonances can be excited at a certain value. The amplitudes are decreasing with the fundamental component of the magnetic flux-density increasing, which presents the fundamental component as a damping for the resonance of the stator system in Figure 5(a). According to (2), the influences of the stator and rotor windings harmonic components are similar. And Figure 5(b) shows that the stator windings harmonic components make 
the amplitudes of the first and third modes increase in the resonance region. That means the harmonic magnetic field of the stator and rotor is the main source of electromagnetic excitation.

5.4. Dynamic Characteristics. In the following figures, diagrams of time history, phase chart, FFT, and Poincaré section are presented for further understanding of the combined internal and principal parametric resonances of the stator system. Based on the parameters in Figures $2-5$, by assuming $\varepsilon \delta_{1}=0.12, \varepsilon \delta_{2}=0.48, \mu=8, B_{\delta}=0.75 \mathrm{~T}$, and $B_{v}=B_{\mu}=0.1 \mathrm{~T}$, Figures $6(\mathrm{a})$ through $6(\mathrm{~d})$ confirm that the system is period-1 motion according to Figure $4(\mathrm{~d})$. The phase chart is a closed circle, and the energy of the fourth mode is much larger than that of the first and third modes. There is only one nonzero point, and the other two zero points coincide with each other in the Poincaré section. That means the stator system is in stable single-frequency and periodic motion. With the damping coefficients reducing from 8 to 6, there are three points in Figure 7(d) and three frequencies in Figure 7(c). The energy of the fourth mode transfers to the first and third modes, but still higher than the other modes. The stator system is in quasiperiod motion. When the damp coefficients reduce from 6 to 2, the energy of the first mode is the highest in Figure 8. And the phase chart presents reciprocating motion in a bounded domain. With the amplitude of electromagnetic excitation increasing to $B_{\delta}=0.5 \mathrm{~T}$ and $B_{v}=B_{\mu}=0.2 \mathrm{~T}$, the energy of all three modes is higher than the former, and the amplitude of the stator system increases. Figures 9(a) through 9(d) confirm the conclusion of Figure 5.

It is essential to point out that when $\varepsilon \delta_{1}=0.52$, there is an additional relation between the first and fourth natural frequencies as $\bar{\omega}_{4} \approx 3 \bar{\omega}_{1}$. But the coupling coefficient $\Gamma_{4111}=$ 0 which means there is only frequency relationship, and no interaction between these two modes. The expression in (11) is still effective. Figures 10(a) through 10(d) show that the stator system presents the period-2 motion.

\section{Conclusion}

The combined internal and principal parametric resonances on the stator system of an asynchronous machine excited by the varying distributed electromagnetic force are investigated in this paper. The stator system is modeled as a continuum coupled by two cylindrical shells. And the distributed electromagnetic force expression with the interaction between mechanical and electromagnetic behavior is given. The numerical simulation results show the influences of mechanical and electromagnetic parameters on the resonances.

When there is a resonance relationship between natural and excitation frequencies, and the corresponding coupling coefficients of modes are nonzero, the forced resonances occur. Simultaneously, in the presence of the resonance relationship of natural frequencies, the combined internal and principal parametric resonances occur and excite three modes. The jump phenomenon is pointed out and the amplitudes of the first and third modes are much higher than the fourth mode. Energy can be transferred directly from the excited fourth mode to the other modes through the internal mechanism. The harmonic magnetic field of the stator and rotor is the main source of electromagnetic excitation. Furthermore, diagrams of time history, phase chart, FFT, and Poincare section are presented to show the complicated dynamic characteristic of the stator system with the response of period-1, quasiperiod, and particularly double period motions which should be avoided in engineering practice.

\section{Conflict of Interests}

The authors declare that there is no conflict of interests regarding the publication of this paper.

\section{Acknowledgment}

This work was supported by a grant from the innovation team training plan of Tianjin colleges (TD12-5043).

\section{References}

[1] C. Wang and J. C. S. Lai, "Vibration analysis of an induction motor," Journal of Sound and Vibration, vol. 224, no. 4, pp. 733756, 1999.

[2] F. Ishibashi, M. Matsushita, S. Noda, and K. Tonoki, "Change of mechanical natural frequencies of induction motor," IEEE Transactions on Industry Applications, vol. 46, no. 3, pp. 922927, 2010.

[3] S. P. Verma and A. Balan, "Experimental investigations on the stators of electrical machines in relation to vibration and noise problem," IEE Proceedings-Electric Power Applications, vol. 145, no. 5, pp. 455-461, 1998.

[4] M. Al Nahlaoui, D. Braunisch, B. Eichinger, S. Kulig, B. Ponick, and U. Werner, "Calculation methods for electromagnetically excited noise in induction motors," in Proceedings of the 1st International Electric Drives Production Conference (EDPC '11), pp. 124-131, Nuremberg, Germany, September 2011.

[5] P. Pellerey, V. Lanfranchi, and G. Friedrich, "Coupled numerical simulation between electromagnetic and structural models. influence of the supply harmonics for synchronous machine vibrations," IEEE Transactions on Magnetics, vol. 48, no. 2, pp. 983-986, 2012.

[6] A. Shahaj and S. D. Garvey, "A possible method for magnetostrictive reduction of vibration in large electrical machines," IEEE Transactions on Magnetics, vol. 47, no. 2, pp. 374-385, 2011.

[7] A. Belahcen, "Magnetoelastic coupling in rotating electrical machines," IEEE Transactions on Magnetics, vol. 41, no. 5, pp. 1624-1627, 2005.

[8] D. Guo, F. Chu, and D. Chen, "The unbalanced magnetic pull and its effects on vibration in a three-phase generator with eccentric rotor," Journal of Sound and Vibration, vol. 254, no. 2, pp. 297-312, 2002.

[9] P. Pennacchi and L. Frosini, "Dynamical behaviour of a three-phase generator due to unbalanced magnetic pull," IEE Proceedings-Electric Power Applications, vol. 152, no. 6, pp. 13891400, 2005.

[10] P. Pennacchi, "Computational model for calculating the dynamical behaviour of generators caused by unbalanced magnetic 
pull and experimental validation," Journal of Sound and Vibration, vol. 312, no. 1, pp. 332-353, 2008.

[11] P. Pennacchi, "Nonlinear effects due to electromechanical interaction in generators with smooth poles," Nonlinear Dynamics, vol. 57, no. 4, pp. 607-622, 2009.

[12] H. Im, H. H. Yoo, and J. Chung, "Dynamic analysis of a BLDC motor with mechanical and electromagnetic interaction due to air gap variation," Journal of Sound and Vibration, vol. 330, no. 8, pp. 1680-1691, 2011.

[13] G. H. Qing, Y. H. Liu, J. J. Qiu, and X. J. Meng, "A semi-analytical method for the free vibration analysis of thick double-shell systems," Finite Elements in Analysis and Design, vol. 42, no. 10, pp. 837-845, 2006.

[14] H. Nguyen, "Simultaneous resonances involving two mode shapes of parametrically-excited rectangular plates," Journal of Sound and Vibration, vol. 332, no. 20, pp. 5103-5114, 2013.

[15] U. H. Hegazy, "Nonlinear vibrations of a thin plate under simultaneous internal and external resonances," Journal of Vibration and Acoustics, Transactions of the ASME, vol. 132, no. 5, Article ID 051004, 2010.

[16] A. H. Nayfeh and D. T. Mook, Nonlinear Oscillations, John Wiley \& Sons, New York, NY, USA, 1979. 

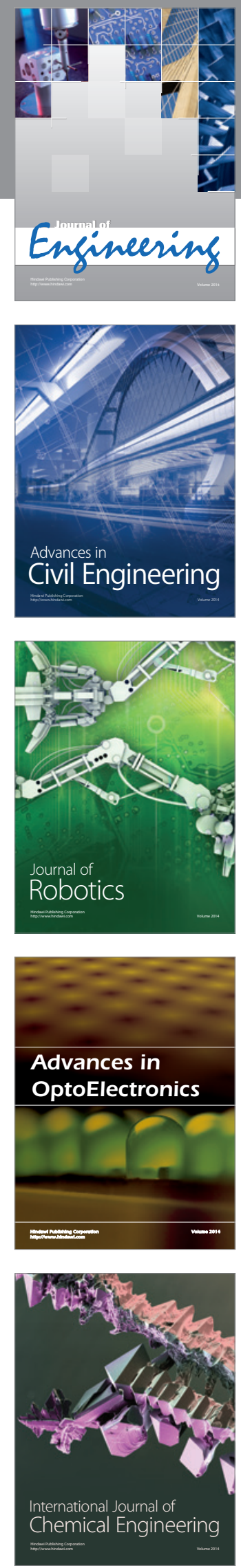

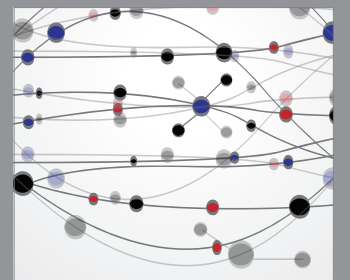

The Scientific World Journal
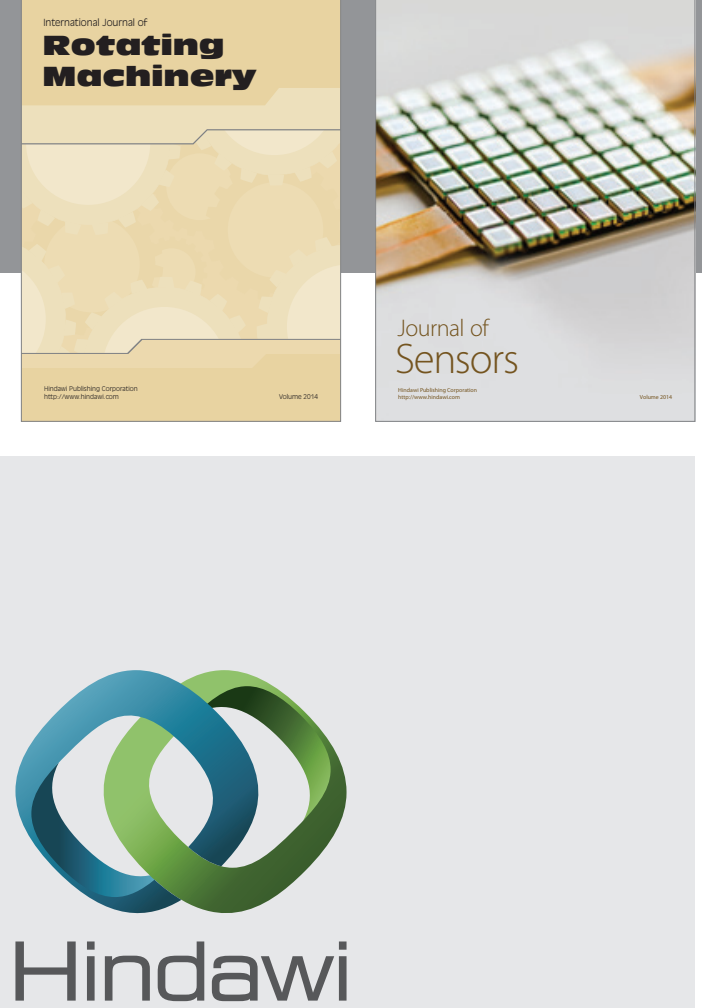

Submit your manuscripts at http://www.hindawi.com
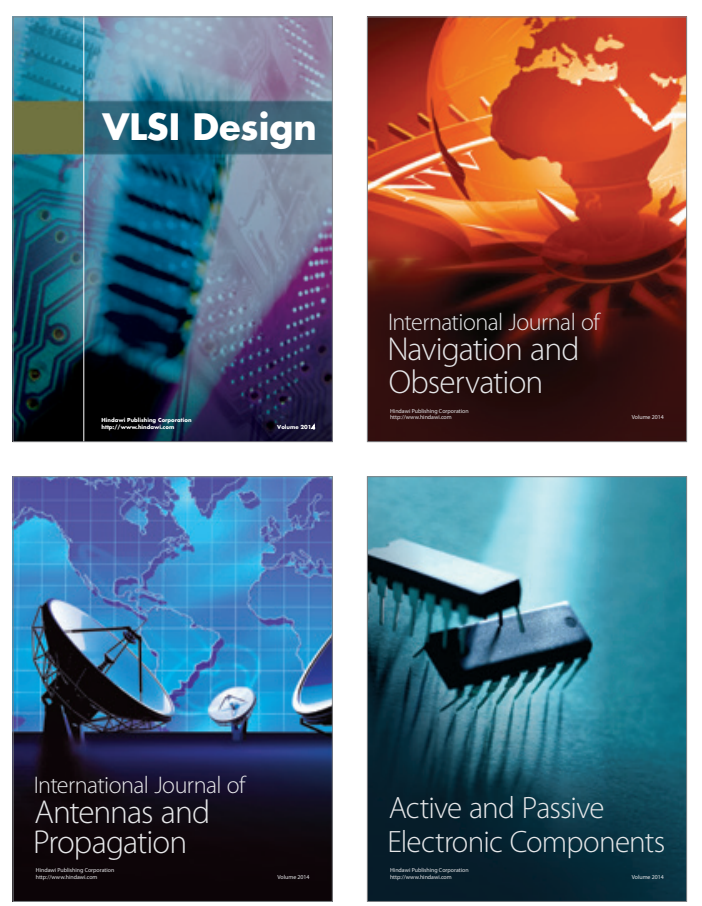
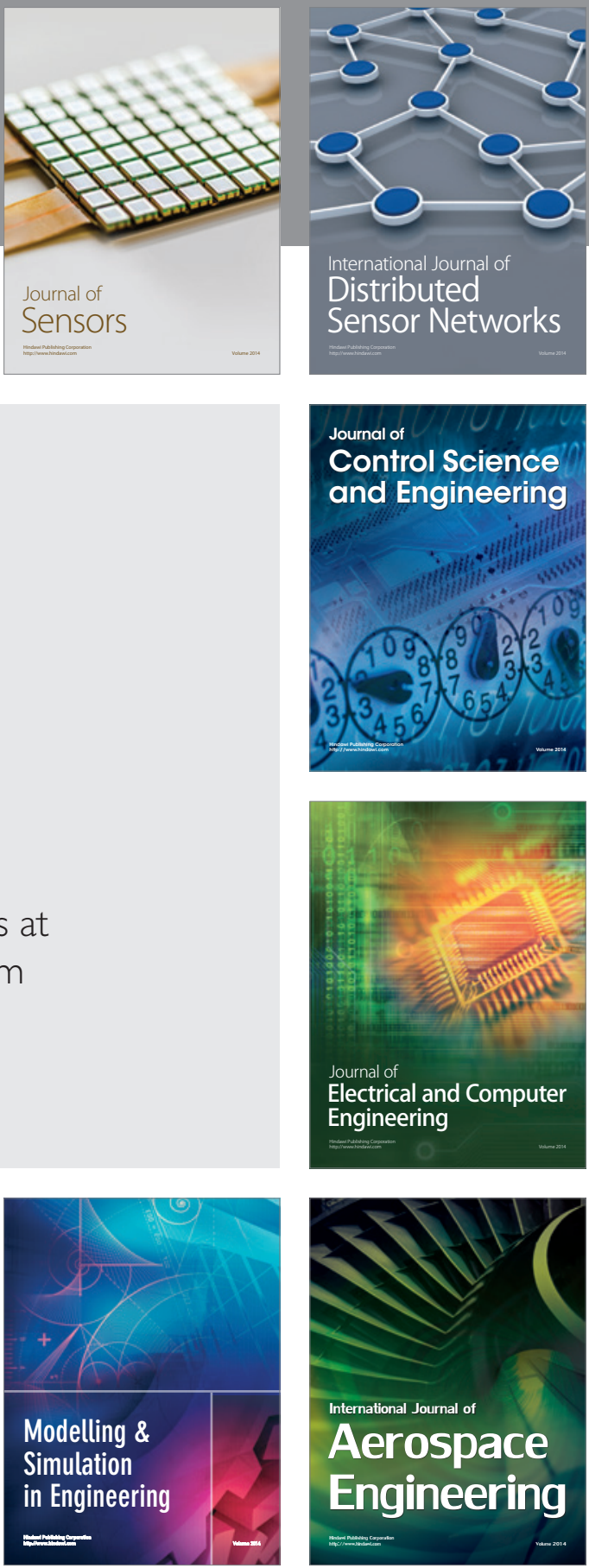

Journal of

Control Science

and Engineering
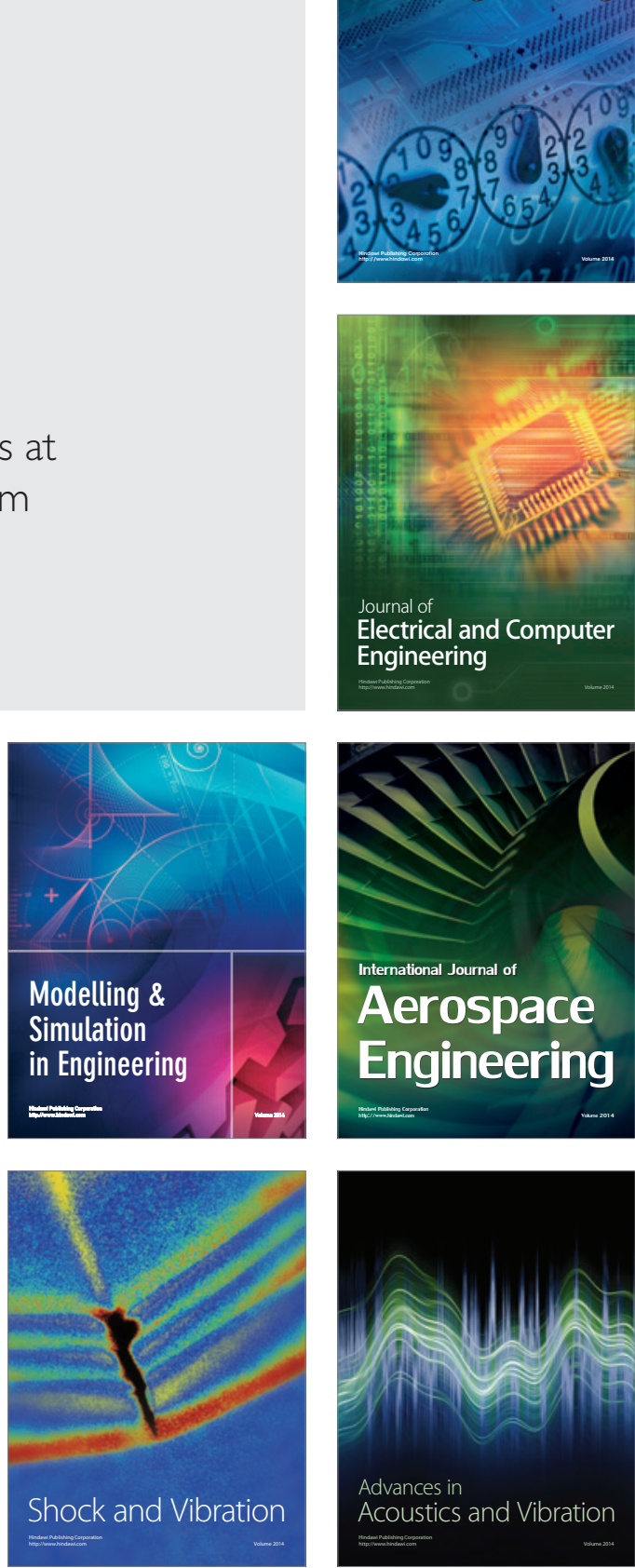